sciendo

International Conference KNOWLEDGE-BASED ORGANIZATION

Vol. XXV

No 3

2019

\title{
USING PERSISTENCE SPECTRUM FOR REALISTIC EXPOSURE ASSESSMENT TO TIME VARYING RADIOFREQUENCY SIGNALS
}

\author{
Annamaria SÂRBU, Simona MICLĂUȘ, Paul BECHET, Iulian BOULEANU \\ “Nicolae Bălcescu” Land Forces Academy, Sibiu, Romania \\ paljanosanna@yahoo.com
}

\begin{abstract}
In this article we propose the use of the persistence spectrum to accurately assess the user's exposure to electromagnetic field emitted by new generation of communication terminals. Persistent display view available within a Real Time Spectrum Analyzer (RTSA) enables a graphical observation of the main statistical parameters of a signal. A simple case study of exposure assessment was conducted by measuring the electric field strength in the close proximity of a mobile phone operated in a LTE-Advanced mobile communication network. The proposed measurement procedure is presented and then applied to a limited number of operating situations (VoIP, Video streaming, File upload, File download). We made use of the persistence spectrum capabilities to directly access the signal strength statistics in time. By this procedure we demonstrate a supplementary facility that enables direct visual tracing of exposure dynamics during real time usage of a specific mobile application.
\end{abstract}

Keywords: LTE-A, persistence spectrum, near field, exposure assessment,

\section{Introduction}

The $4^{\text {th }}$ generation $(4 \mathrm{G})$ mobile communication technology was rapidly deployed to consumers as a response to their increasing demand for faster and better access to information. Today engineers are developing the $5^{\text {th }}$ generation communication networking by targeting not only high data rates but also energy saving, cost reduction or increased system capacity. Long Term Evolution (LTE) was developed as a mobile communication standard aimed not only at increased end user data rates but also at increased system capacity. The standard is part of the fourth generation technology (4G) communication standards. LTE uses orthogonal frequency-division multiple access (OFDMA) technique for downlink communication. A pre-coded version of the same modulation technique, single-carrier frequency-division multiple access (SC-FDMA) technique is used for uplink communication. LTE Advanced (LTE-A) was the follow-up standard whose main improvement consists in the use of multiple antennas (MIMO) on both the transmitter and the receiver. In addition, it implements Carrier Aggregation (CA) enabling a device to communicate on several different $4 \mathrm{G}$ signals at once [1].

With signals belonging to new generation communication era behaving as stochastic like processes, electromagnetic fields (EMF) exposure assessment studies became more and more complex. Public concerns are being raised as the rate of these studies can hardly follow the rate of modern technological development.

The majority of the studies with exposure assessment goals deal with EMF emitted in downlink by mobile communication base station antennas [2]. One of the few studies 
focused on mobile terminals is based on network measurements and concludes that the mean output powers of a $4 \mathrm{G}$ mobile device is less than $1 \%$ of the maximum available emitted power [3]. Even so, there are even less studies conducted on mobile terminals in real life operating situations [4]. The results of these studies strongly motivate the need for realistic exposure assessment, with common daily routine operating scenarios.

New generation communication signals are characterized by sporadic or even random energy occurrence. The use of a traditional sweep spectrum analyzer makes it difficult to show all events in a signal especially for modern techniques such as frequency hopping spread spectrum or cognitive radio. This drawback was eliminated in real time spectrum analyzers (RTSA) capable of providing full information on the available real time bandwidth (RTBW). This is done by overlapping Fast Fourier Transforms (FFT) combined with high processing power that gives a high probability of intercept (POI) even in extremely dense environments.

The persistence display of a RTSA gives information not only about the amplitude of the measured signal at a given moment in time but also on the frequency of occurrence of that amplitude level over an observation time.
The persistence display mode gives quick access to a power versus time histogram which conveys both average and standard deviation of a set of measurements.

Following some previous work of ours[5,6] this article investigates the capabilities of a persistence spectrum display available in RTS As for providing information about the user's exposure to EMF for a selection of real life operating situations.

\section{Materials and methods}

With the help of a small size electric field probe model Aaronia PBS-E1 we have measured the near field radiated power ofa phone model Huawei P10 Lite WAS-LX1 (further referenced as UE) operated in the Vodafone Ro network. The UE was using the $4 \mathrm{G}+$ (LTE-A) communication network while running four different types of mobile applications: VoIP, Streaming (Youtube $1080 \mathrm{p}$ video quality), File download and File upload. The Whats App Android application installed on the UE was used for VoIPwhile all other applications were launched on a PC browser having the internet connection USB tethered by the mobile phone. The USB tethering was chosen to avoid interference with the UE during a set of measurements and to keep the UE battery level at $100 \%$. A picture of the experimental setup is presented in figure 1 .

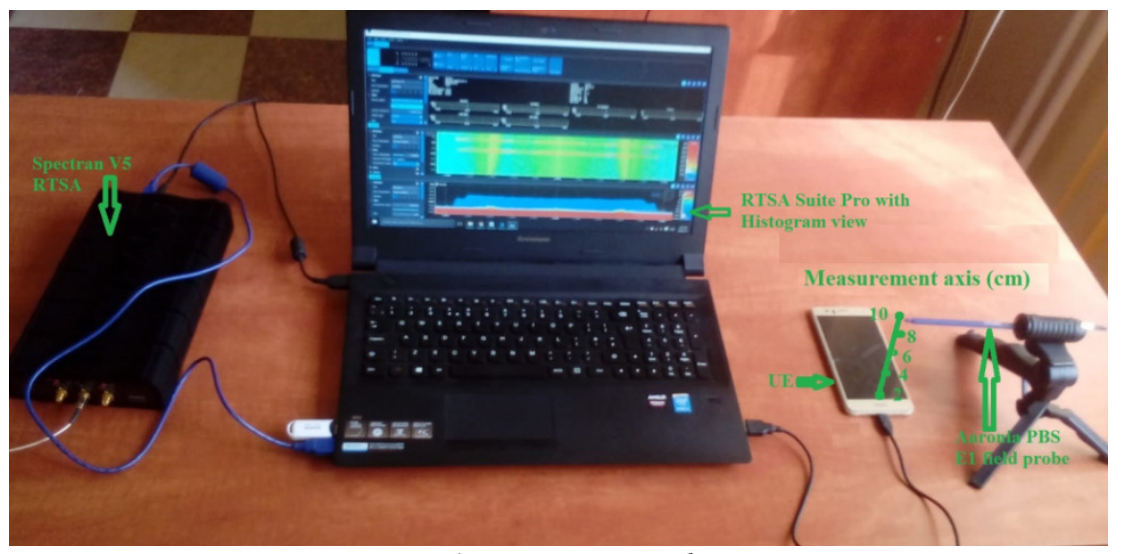

Figure 1: Experimental setup

Measurements of the E-field strength were taken on a single at five equally spaced points situated at 2,4,6,8 and $10 \mathrm{~cm}$ from the UE surface. Measurement duration was 
chosen to be 1 minute for each point. During this time the selected application service was solely active. The axis positioning on the mobile phone was chosen after preliminary scanning to be at the observed "hot spot." The near field probe positioning was chosen to be on one axis only because previous measurements of the same UE demonstrate that the majority of the electric field strength was measured on this direction [6]. The probe was connected by a $3 \mathrm{~m}$ SMA cable to the Spectran HF-80120 V5 X RTSA capable of providing up to $88 \mathrm{MHz}$ RTBW. The measured power levels $(\mathrm{dBm})$ were transformed to electric field strength $(\mathrm{V} / \mathrm{m})$ by using the probe's power converter file. Detailed explanations of general LTE-A characteristics, channel frequency and specific observation at the measurement site can be found in [6].

The Aaronia RTSA Suite Pro software [7] with the spectrum, spectrogram and histogram blocks was used for measurements. The resolution bandwidth (RBW) of the spectrum analyzer was set to $100 \mathrm{kHz}$ to be smaller than the $180 \mathrm{kHz}$ bandwidth specified in the LTE-A standard. The RTBW span was set to $22 \mathrm{MHz}$ to cover the maximum possible LTE-A uplink bandwidth of $20 \mathrm{MHz}$. The number of FFT bins was set to 224 as it provides the same frequency resolution as the set RBW $\sim 100$ $\mathrm{kHz}$ ). With the above presented settings the sample duration was $68 \mathrm{~ns}$ and the calculated POI was $15 \mu \mathrm{s}$. The values of the sample duration and POI were displayed by the signal analyzer for the presented measurement settings.

As compared to a classic spectrum view, the persistent display of a RTSA, often called histogram view, adds information based on signal variation - in time. Signals that were active for a short time but are not currently present in the environment can be seen and measured as they are displayed by a trace. The normalized cumulative frequency of the signal amplitude over time is calculated for each frequency bin. The main settings used for the histogram block were $100 \%$ persistence factor and 128 discrete power level bins in the dynamic range starting from $-85 \mathrm{dBm}$ to $4 \mathrm{dBm}$. The real time bandwidth of the analyzer was divided into 224 frequency bins, whereas the dynamic range was divided into 128 power level bins. These settings were chosen to ensure a resolution of $180 \mathrm{kHz}$ bandwidth $/ 1 \mathrm{dBm}$ power level bin. The persistence display was cleared before each measurement start for each of the measurement points.

The measured uplink EARFACN channel is shared by users of the same LTE-A cell. Figure 2 presents two comparative cases of estimated frequency occupancy after 1minute monitoring.

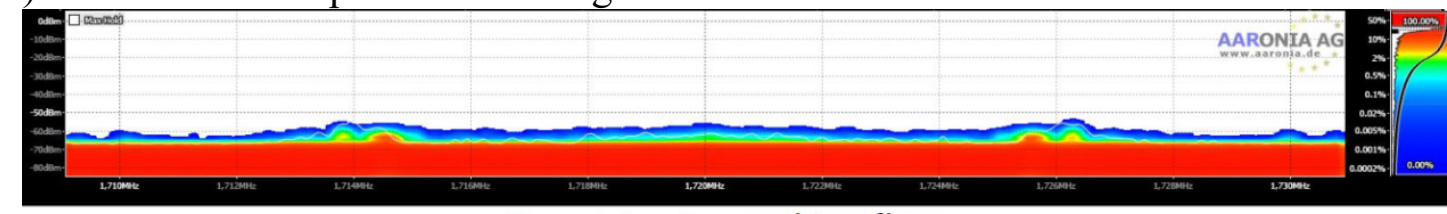

a. Uncrowded spectrum- persistence dispay

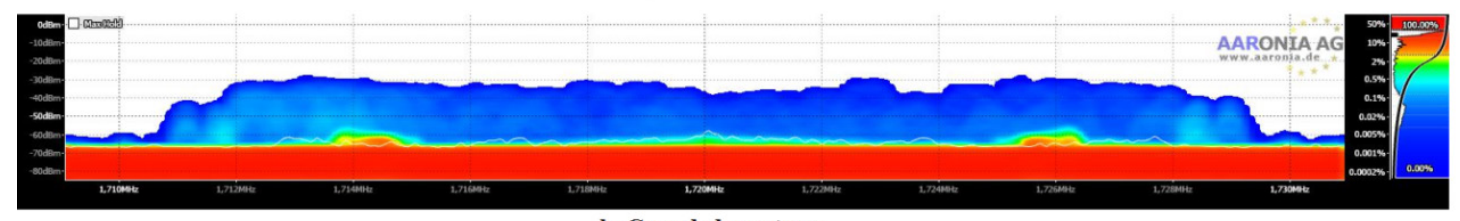

b. Crowded spectrum

Figure2: Frequency occupancy estimation

Figure $2 \mathrm{a}$ was taken at early hours when no other users were present in the environment (building and surroundings) while figure $2 \mathrm{~b}$ reveals a "crowded" frequency bandwidth. In order to evaluate user exposure, first we have to make sure that the measured signal 
is emitted by the device under test only. This assumption was considered suitable because measurements took place in the near field of the UE antenna at early hours characterized by low channel occupancy (figure 2b). By using persistence spectrum measurements, the user can enable direct view of power versus time histogram. Moreover, data can be saved and further statistical processing can be applied.

\section{Results and discussion}

The global normalized histograms of the 20 $\mathrm{MHz}$ channel bandwidth are presented in figure 3 for all 4 applications measured at 2 selected distances from the UE (2 respectively $10 \mathrm{~cm}$ ). The vertical axis represents the percent of time the signal spent at the given power level/field strength from the entire measurement time.

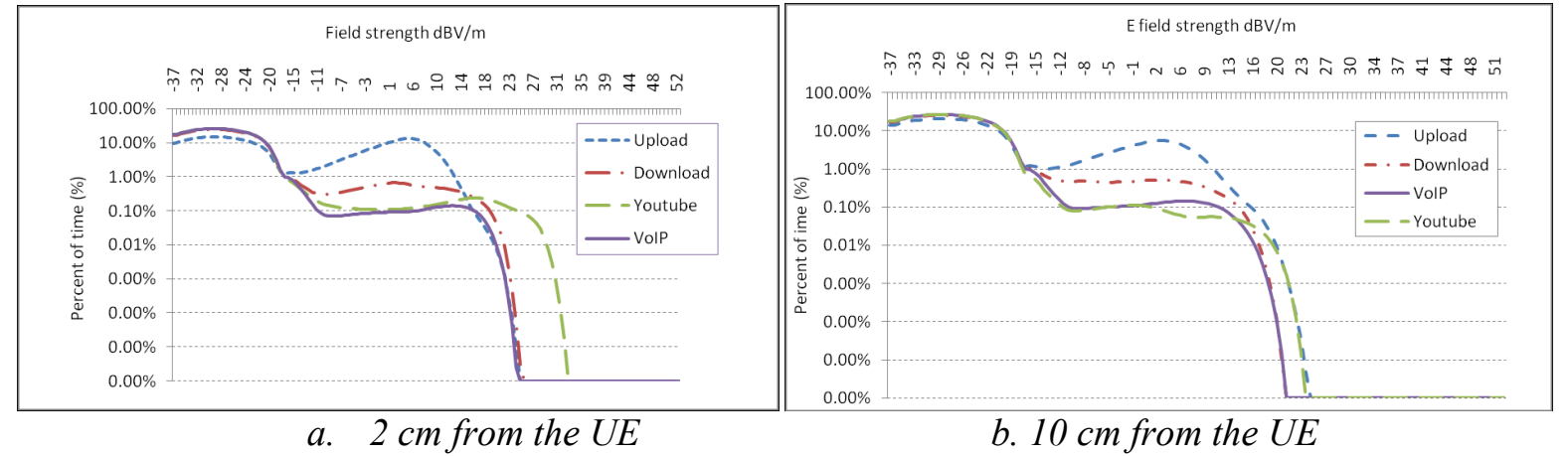

Figure 3: Percentage of time spent at bined field strength value for the entire $20 \mathrm{MHz} L T E-A$ bandwidth -all tested application services

The two peaks visible for all traces presented in figure 3 can be assigned to the noise and signal samples respectively [8]. Channel bandwidth noise was shortest for upload application service while all other have similar channel noise durations (first visible peak in figure 3 a corresponding to $30 \mathrm{dBV} / \mathrm{m})$. If user exposure is of interest, the second peak has to be examined as it belongs to the interest signal samples. One can observe that highest user exposure is expected for upload application services followed by download, streaming and VoIP. The service ranking was maintained for all measurement distances. Similar results were obtained for all distances considered and in figure $3 \mathrm{~b}$ authors chose to present results for $10 \mathrm{~cm}$ measurement distance.

In figure 4 the persistence displays obtained after 1-minute measurement time at $2 \mathrm{~cm}$ from the UE surface are presented for the four tested applications services.

From the persistence spectrum analysis, it is visible which parts of the $20 \mathrm{MHz}$ bandwidth are allocated to the UE. Side band subcarriers are much more visible for both video streaming and file download, while entire $20 \mathrm{MHz}$ bandwidth is used when file upload. Based on this representations, in figure $5 \mathrm{a}$ the normalized histograms of the entire $20 \mathrm{MHz}$ maximum channel bandwidth are presented comparatively with the histograms of the 2 $\mathrm{MHz}$ occupied channels (visible in figure 4 $\mathrm{b}$ and c) for video streaming (YouTube) and File download. As expected, in the case of the $2 \mathrm{MHz}$ occupied channel the trace is generally pushed to the right. This means higher E field strength values as compared to those present in the traces of the $20 \mathrm{MHz}$ channel bandwidths are appearing. In addition, the second peak of the histogram representation is much more visible for the $2 \mathrm{MHz}$ selected channels. This is explained by the fact that the signal levels present in the $2 \mathrm{MHz}$ wide channel become more prominent from noise samples.

Figure $5 \mathrm{~b}$ presents the normalized histogram obtained for VoIP application service measured at different distances from the UE. VoIP service was chosen as it 
represents a real time application service and is characterized by the most constant signal levels. As expected, because measurements were performed in the near field, E field strength does not decrease with distance. Two peaks are visible at 6 $\mathrm{cm}$ respectively $10 \mathrm{~cm}$ distance from the UE.
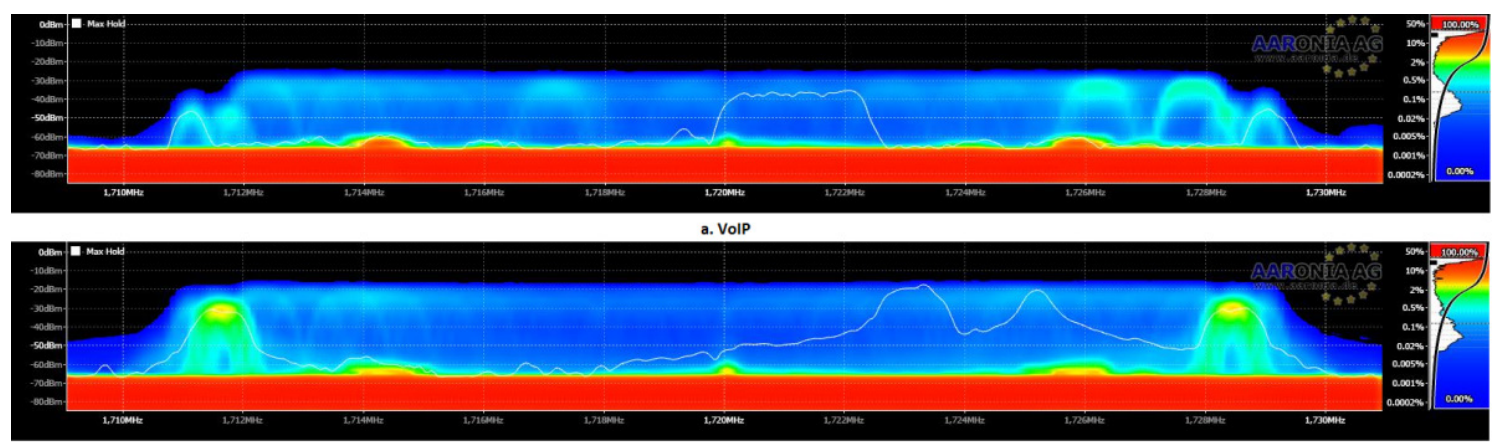

b. Youtube

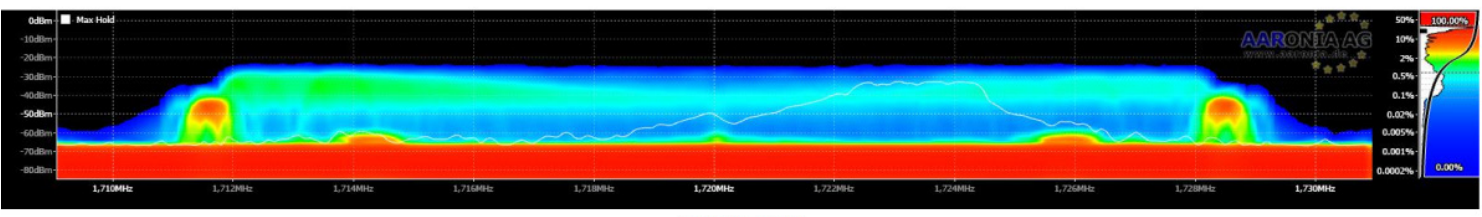

c. File download

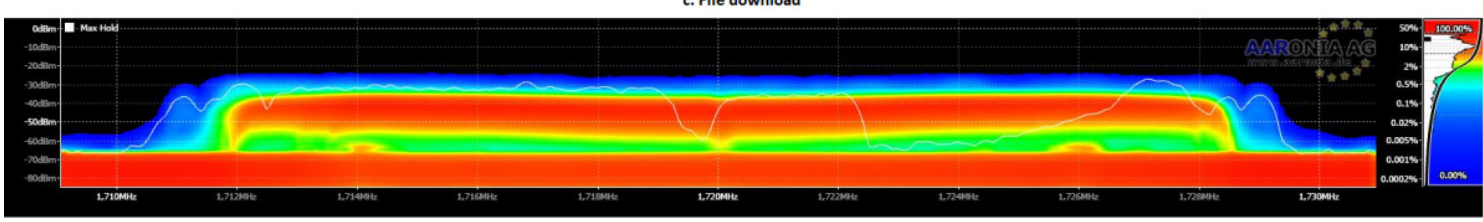

d. File upload

Figure 4: Persistence spectrum for all application services measured at $2 \mathrm{~cm}$ from the UE

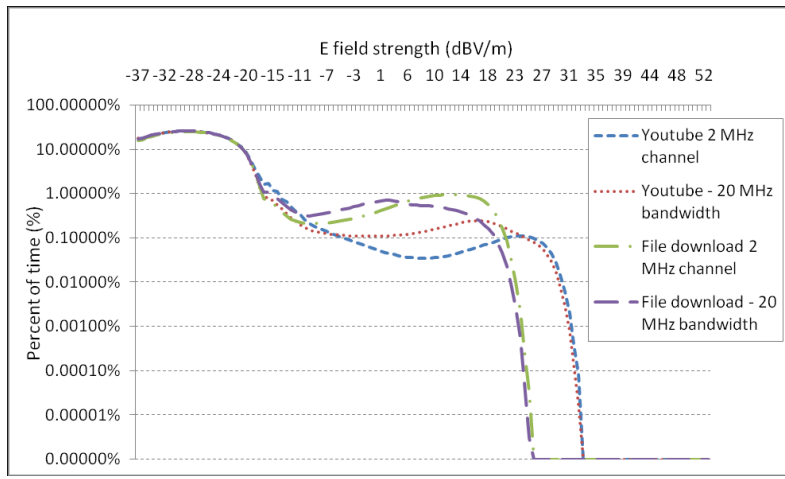

Figure 5a Normalized histograms: comparison between time distribution of binned E-field levelson the entire $20 \mathrm{MHz} L T E-A$ and on a 2 $\mathrm{MHz}$ occupied channel Figure $5 b$ Normalized

\section{Conclusions}

In this paper we have applied persistence spectrum measurements with the aim of obtaining a realistic image of user exposure to LTE-A uplink signals in its dynamics by considering four operating scenarios. Results presented here demonstrate that persistence spectrum can be used to obtain

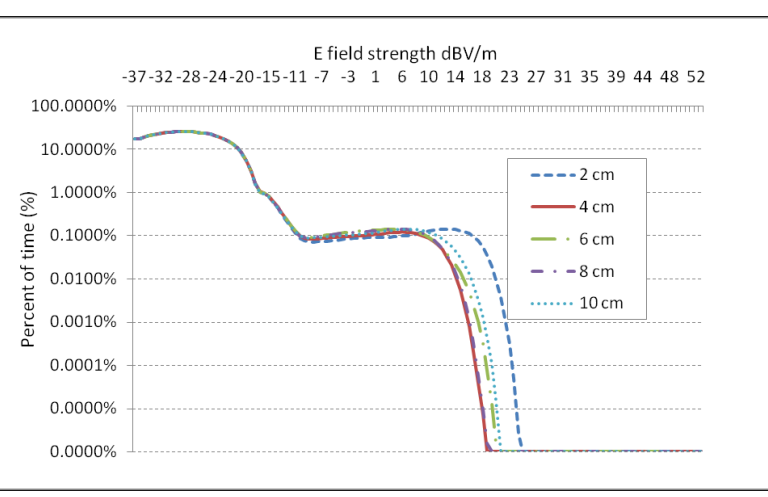

histograms of time distribution ofbinned E-field levels for VoIP application service measured at different distances from the UE.

a first visual on the exposure evolution in time. Real time measurements features, such as Complementary Cumulative Distribution Function or Probability Density Function, can provide a more indepth analysis of the signal statistical behavior. However, the use of persistence spectrum has the advantage of being a 
direct visual instrument that can be used for rapid description of main signal evolution in time, frequency and power domains. In addition, the power versus time histograms can be used for detailed analysis and can provide information on both the interest signal and the noise in the measured channel (as amplitude and duration).
By applying the proposed method, we emphasize the advantage obtained by using real time spectrum analysis for EMF exposure evaluation purposes. Direct access to parameters obtained after automatic statistical processing makes persistence spectrum a very useful tool for exposure assessment technicians.

\section{References}

[1] E. Dahlman, S. Parkvall, J. Skold, “ 4G LTE/LTE-Advanced for Mobile Broadband, ISBN:978-0-12-385489-6.

[2] Joseph W, Verloock L, Goeminne F, Vermeeren G, Martens L., In situ LTE exposure of the general public: Characterization and extrapolation, Bioelectromagnetics, 2012 September; Vol.33(6):466-75

[3] Joshi, P., Colombi, D., Thors, B., Larsson, L.-E., Tornevik, C., Output Power Levels of 4G User Equipment and Implications on Realistic RF EMF Exposure Assessments, IEEE Access, 2017, Vol. 5, pp. 4545-4550

[4] Jovanovic D., Bragard G., Picard D. and Chauvin S., Mobile telephones: A comparison of radiated power between $3 \mathrm{G}$ VoIP calls and $3 \mathrm{G}$ VoCS calls, Journal of Exposure Science and Environmental Epidemiology (2015) 25, pp. 80-83

[5] Paljanos, A., Miclăuș, S., Bechet, P., \&Munteanu, C. (2016). Assessment of mobile phone user exposure to UMTS and LTE signals: comparative near-field radiated power levels for various data and voice application services. Journal of Electromagnetic Waves and Applications, 30(9), 1101-1115. https://doi.org/ 10.1080/09205071.2016.1167634

[6] AnnamariaSârbu, Andrei Bechet, Titus Bălan, Dan Robu, Paul Bechet, Simona Miclăuş, Using CCDF statistics for characterizing the radiated power dynamics in the near field of a mobile phone operating in $3 \mathrm{G}+$ and $4 \mathrm{G}+$ communication standards, Measurement, Volume 134,2019,Pages 874-887,ISSN 0263-2241

[7] http://www.aaronia.com/rtsa-manual/en/Content/Home.htm

[8] Datla, Dinesh \& M Wyglinski, Alexander \& Minden, Gary. (2007). A Statistical Approach to Spectrum Measurement Processing 